\title{
Impact of lymph node staging systems in predicting outcome in patients with ampullary cancer
}

\author{
Sundeep Singh Saluja, Pramod Kumar Mishra, Shashi Kiran, Harsh J. Shah, Sandip Chandrasekar, \\ Mohammed Nayeem, Aditya Sharma, and Vaibhav Kumar Varshney \\ Department of Gastrointestinal Surgery, Govind Ballabh Pant Institute of \\ Postgraduate Medical Education and Research, New Delhi, India
}

\begin{abstract}
Backgrounds/Aims: Lymph node (LN) metastasis though, is a poor prognostic factor for ampullary carcinoma (APC), the impact of Lymph node ratio (LNR) and Logarithm odds of positive lymph node (LODDS) in the long-term survival remains controversial. We evaluated the factors affecting the long-term outcome in APC patients with emphasis on LNR and LODDS. Methods: The prospectively collected data of 198 patients who underwent pancreatoduodenectomy for APC was analyzed after excluding 12 patients for various reasons. Factors affecting Disease specific survival (DSS) and Recurrence free survival (RFS) were analyzed with special reference to LN positivity, LNR and LODDS. Results: Out of 186, $117(62.9 \%)$ patients were alive at a median follow-up of 39.5 months and $72(38.7 \%)$ developed recurrence. The overall 5-year DSS was $59.3 \%$ \& RFS $54.9 \%$. Univariate analysis showed T-stage, tumor differentiation, perineural invasion, LN positivity, LNR and LODDS was significantly affected DSS and RFS. On multivariate analysis, perineural invasion, LN positivity, LNR and LODDS lost its significance for DSS and RFS. AUC for prediction of DSS and RFS for LNR was $0.654(p<0.001) \& 0.629(p=0.003)$ respectively and for LODDS, it was $0.697(p<0.001) \&$ $0.677(p=0.001)$ respectively. Sensitivity and specificity of LNR $(0.1)$ for DSS were $37.7 \% \& 83.8 \%$ and for RFS were $36.1 \%$ \& 83.3\%; for LODDS (-1.00), sensitivity and specificity for DSS was $62.3 \%$ and $67.5 \%$ and for RFS it was $59.7 \%$ and $66.7 \%$ respectively. Conclusions: LNR and LODDS although independently seem to affect the RFS and DSS, albeit have a low sensitivity and specificity in predicting DSS and RFS. (Ann Hepatobiliary Pancreat Surg 2020;24: 484-495)
\end{abstract}

Key Words: Periampullary; Pancreatoduodenectomy; Lymph node ratio; LODDS; Survival; Long term

\section{INTRODUCTION}

Traditionally, periampullary carcinoma (PACA) comprised of four heterogeneous tumors arising from ampulla, duodenum, pancreatic and distal bile duct. Among them, ampullary carcinoma, is the second most common variant of PACA and accounts for $\sim 0.5 \%$ of all neoplasms arising from the gastrointestinal tract. ${ }^{1}$ Surgical resection remains the mainstay of treatment for patients with periampullary carcinoma (PACA). The long-term survival following resection of PACA is governed by a number of factors, such as the tumor $(\mathrm{T})$ differentiation, $\mathrm{T}$ stage, lymph node status, margin status and perineural invasion. However, these factors are reported to have varied importance among different site in periampullary tumor especially lymph node status.

Lymph node metastasis is considered to be one of the important predictors of survival in various GI malignan$\mathrm{cy}^{2-7}$ Survival analysis can have a bias if the lymph node estimate in specimen is inaccurate. Apart from lymph node positivity, total number of $\mathrm{LN}$ retrieved, number of positive lymph nodes, lymph node ratio (LNR-ratio between number of positive LN and number of LN retrieved) and logarithm odds of positive lymph node (LODDS) have been suggested to have a better prognostic significance in pancreatic and periampullary carcinoma. ${ }^{4,7-9}$

LNR has been suggested as a prognostic marker for various cancers namely esophageal, gastric, colonic, rec-

Received: January 29, 2020; Revised: June 8, 2020; Accepted: June 9, 2020

Corresponding author: Sundeep Singh Saluja

Department of Gastrointestinal Surgery, Govind Ballabh Pant Institute of Postgraduate Medical Education and Research, 1, Jawaharlal Nehru Marg, New Delhi 110002, India

Tel: +919718599259, E-mail: sundeepsaluja@yahoo.co.in

Copyright (C) 2020 by The Korean Association of Hepato-Biliary-Pancreatic Surgery

This is an Open Access article distributed under the terms of the Creative Commons Attribution Non-Commercial License (http://creativecommons.org/ licenses/by-nc/4.0) which permits unrestricted non-commercial use, distribution, and reproduction in any medium, provided the original work is properly cited. Annals of Hepato-Biliary-Pancreatic Surgery • pISSN: 2508-5778 - elSSN: 2508-5859 
tal, pancreatic as well as periampullary malignancies. ${ }^{2-11}$ However studies on ampullary tumor have suggested that number of lymph nodes involved may predict survival better than LNR while others support LNR as better prognostic marker. ${ }^{12-14}$ Caution needs to be exercised considering the alacrity with which the proponents of LNR are proliferating. Log odds of metastatic LN (LODDS) as a LN staging method for prognostic marker was considered as an alternative since it took into consideration the status of probability of negative nodes patients also. ${ }^{15-17}$

The aim of our study was to critically evaluate the role of various factors with particular emphasis on LN staging methods including LNR and LODDS for prognostication of patients undergoing pancreatoduodenectomy for ampullary adenocarcinoma.

\section{MATERIALS AND METHODS}

We analyzed the data of all patients with periampullary carcinoma treated at our tertiary care referral center from January 2004 to December 2018 from a prospectively maintained database.

\section{Inclusion criteria}

All patients undergoing pancreatoduodenectomy for suspected / proven periampullary tumor were included in the study.

\section{Exclusion criteria}

The patients who had a final histopathology other than adenocarcinoma and those with distal CBD, duodenal and pancreatic variant of Periampullary carcinoma were excluded from the study. R1 resections were also excluded to avoid potential bias from inclusion of probable locally advanced tumors which were left inadvertently with positive margins.

\section{Work-up and operative procedure}

All patients underwent contrast enhanced CT scan for pre-operative staging. Pre-operative biliary drainage was done only in patients with serum bilirubin level exceeding 15 $\mathrm{mg} \%$ or if there was an evidence of cholangitis. All patients underwent pancreatoduodenectomy and reconstruction of pancreatico-jejunostomy using by modified Blumgart's technique. ${ }^{18}$ The extent of lymph node dissection included removal of pancreaticoduodenal, pericholedochal, periportal, along hepatic artery and lymph nodes to the right of coeliac and superior mesenteric artery. All surgeries were performed under direct supervision of two senior surgeons (PM, SS). Resection margins in the specimen at the level of bile duct, pancreatic duct, superior mesenteric artery and superior mesenteric vein were inked/marked before sending for histopathology. Resected specimens were analyzed for the location, size and differentiation of the tumor, perineural invasion (PNI), status of the lymph nodal involvement and resection margins.

Follow-up protocol included out-patient visits every 3 months in the $1^{\text {st }}$ year, every 6 months for next 2 years and yearly thereafter. At each follow up complete physical examination along with blood biochemistry and ultrasound abdomen were performed. The CT scan of the abdomen was performed at 1-year and then at every 2-year intervals or when clinically indicated.

\section{Parameters analyzed}

The demographic profile and histopathological details including tumor differentiation, perineural invasion, margin status, $\mathrm{T}$ stage, total number of lymph nodes retrieved and the number of positive lymph nodes were reviewed and analyzed. Lymph nodes positivity was divided into N0, N1 and N2 as per AJCC $8^{\text {th }}$ edition.

LNR was calculated by dividing the number of positive lymph nodes by the total number of lymph nodes retrieved. LNR was assessed as both continuous and categorical variable.

a) LNR assessment as categorical: LNR were categorized as: I- $\leq 0.05$; II- $>0.05 / \leq 0.1$; III- $>0.1 / \leq 0.2$; IV$>0.2 / \leq 0.3$.

b) LNR assessment as continuous variable: ROC curves were plotted for LNR against disease specific survival (DSS) and recurrence free survival (RFS). Area under curve, sensitivity and specificity were determined.

Logs odd of positive nodes (LODDS) was calculated by $\log ($ pnod $+0.5 /$ tnod-pnod +0.5$)$ where pnod is number of positive nodes while tnod is total number of examined nodes and 0.5 is added to numerator and denominator to avoid infinite number. Cut-off of LODDS were classified according in groups: LODDS1: LODDS $<-3$; LODDS2: $-3 \leq$ LODDS $<-2$; LODDS3: $-2 \leq$ LODDS $<-1$; LODDS4: $-1 \leq$ LODDS $<0$; LODDS5: $0 \leq$ LODDS $<1{ }^{16}$ 
Recurrences were classified as local (nodal / tumor bed), distant (liver, peritoneum, non-regional lymph node, systemic) or both.

Survival data were obtained either from the date of the last out-patient visit or via personal communication with the patients. Deaths occurring up to $30^{\text {th }}$ post-operative day were considered post-operative mortality and were excluded from the survival analysis. Disease specific survival was defined as the time period from start of treatment till the time of death. Recurrence free survival was defined as the time period from start of treatment till the time at which recurrence was detected.

\section{Statistical analysis}

Categorical variables were analyzed by Chi-square test and continuous variables with t-test or Mann-Whitney $U$ test, where appropriate. Survival probabilities were calculated using Kaplan-Meier method and compared by Logrank test. Cox regression was used for multivariate analy- sis and hazard ratios (HR) were calculated. Results were considered significant if the $p$-value was less than 0.05 . Statistical analysis was performed using SPSS software (version 23 for Mac, IBM Inc., Armonk, NY, USA). Area under curve (AUC) between 0.7-0.8 was considered good predictor while AUC below 0.7 as poor predictor.

\section{RESULTS}

Five hundred and sixty six patients with periampullary carcinoma were admitted for definitive management during the study period. Among these, 364 (64.3\%) patients underwent pancreatoduodenectomy while 202 (35.7\%) were deemed inoperable or unresectable (metastatic disease or locally advanced tumors). The final histopathology report revealed adenocarcinoma in 321 patients while 43 patients who had other etiologies were excluded. Among 321 patients, 123 patients [Distal CBD $(\mathrm{n}=60,18.7 \%)$, duodenal $(\mathrm{n}=37,11.5 \%)$ and pancreatic $(\mathrm{n}=26,8.1 \%)]$ variant of

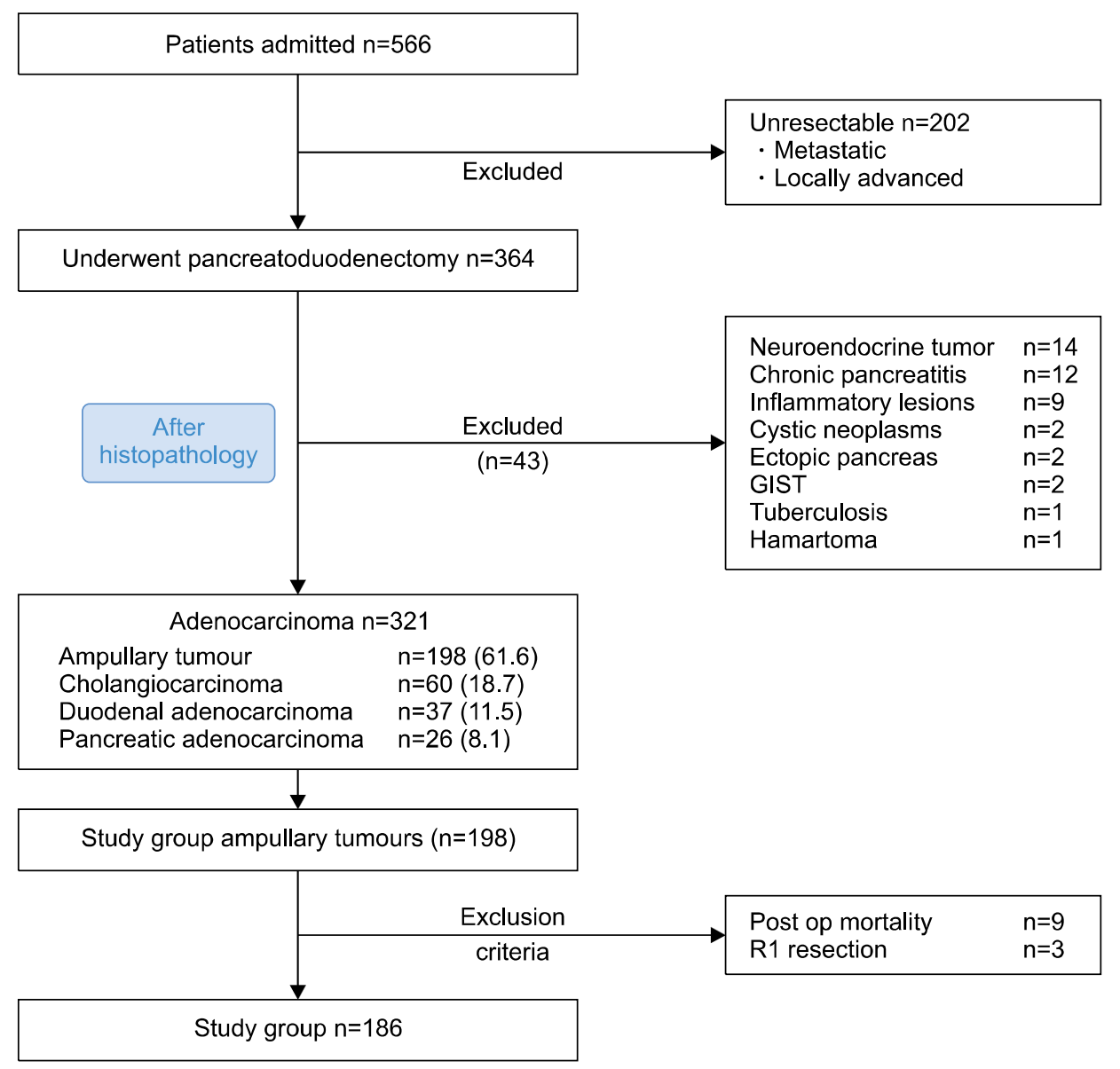

Fig. 1. Patient profile with inclusion and exclusion criteria. 
PACA were excluded while one hundred ninety eight patients with ampullary tumor were reviewed. Patients with peri-operative deaths $(n=9)$ and $R 1$ resection $(n=3)$ were also excluded from analysis. One hundred and eighty six patients fulfilled the study criteria and were included in the study (Fig. 1).

\section{Demographic and pathological findings}

The median age of ampullary tumor was 50 (25-78) years with male is to female ratio was 1.55 . None of the patients received neoadjuvant therapy. Perineural invasion was detected in $45(24 \%)$ patients. The poorly differentiated carcinoma comprised of $7 \%(n=13)$, whereas $47 \%$ patients had T3-4 tumors (Table 1). The lymph node positivity was found in 77 (41\%) cases. The median number of lymph nodes examined was 15 . The median number of LN retrieved was 14.5 among LN negative patients, while it was 16.5 for $\mathrm{LN}$ positive patients.

\section{Survival}

Of 186 patients who underwent resection, 117 patients $(62.9 \%)$ were alive at the time of the last follow-up. Median follow-up period was 39.5 months (4-168 months). The 3-year and 5-year DSS was $72.1 \%$ and $59.3 \%$, while RFS was $66 \%$ and $54.9 \%$ respectively. Overall 72 patients (38.7\%) developed recurrence during the follow up (Table 2). Among these, 51 patients had distant metastasis alone [most common being liver ( $\mathrm{n}=40,55.5 \%)$, followed by peritoneum $(n=6,8.3 \%)$, lung $(n=3,4.2 \%)$ and brain $(n=2$, 2.8\%)], 12 patients had local recurrence alone (16.7\%), while 9 patients had recurrence at both sites (12.5\%).

\section{Factors effecting disease specific survival}

Univariate analysis revealed $\mathrm{T}$ stage, tumor differentiation, perineural invasion, lymph node positivity, LNR $(0.05,0.1)$, number of positive lymph nodes $(0 / 1-3 />3)$, and LODDS as the significant risk factors influencing DSS (Table 3). Survival curves stratified by T-stage, PNI, LODDS, differentiation, LNR (0.1) and number of positive lymph nodes (AJCC $8^{\text {th }}$ ed.) are shown in Fig. 2. Tumor stage and differentiation were the significant factors on multivariate analysis (Table 3). AUC of LNR and LODDS for predicting DSS (Fig. 2) was $0.654(p<0.001)$ and 0.697 ( $p=0.001)$ respectively.

The sensitivity and the specificity for various cut-off
Table 1. Demographic and histopathological characteristics of patient's undergone resection for ampullary adenocarcinoma

\begin{tabular}{|c|c|}
\hline Parameters & $\mathrm{n}=186(\%)$ \\
\hline \multicolumn{2}{|l|}{ Age (years) } \\
\hline$\leq 60$ & $158(85)$ \\
\hline$>60$ & $28(15)$ \\
\hline \multicolumn{2}{|l|}{ Sex } \\
\hline Male & $113(61)$ \\
\hline Female & $73(39)$ \\
\hline \multicolumn{2}{|l|}{$\mathrm{T}$ stage } \\
\hline $\mathrm{T} 1, \mathrm{~T} 2$ & $98(53)$ \\
\hline T3, T4 & $88(47)$ \\
\hline \multicolumn{2}{|l|}{ Differentiation } \\
\hline Well differentiated & $85(46)$ \\
\hline $\begin{array}{l}\text { Moderately or } \\
\text { poorly differentiated }\end{array}$ & $101(54)$ \\
\hline \multicolumn{2}{|l|}{ Perineural invasion } \\
\hline Negative & $141(76)$ \\
\hline Positive & $45(24)$ \\
\hline \multicolumn{2}{|l|}{ Lymph node } \\
\hline Negative & $109(59)$ \\
\hline Positive & $77(41)$ \\
\hline \multicolumn{2}{|c|}{ Lymph node categorization (AJCC 8th) } \\
\hline 0 & $109(59)$ \\
\hline $1-3$ & $58(31)$ \\
\hline$>3$ & $19(10)$ \\
\hline \multicolumn{2}{|l|}{ Lymph node retrieved } \\
\hline$(\leq 12 />12)$ & $71(38) / 115(62)$ \\
\hline$(\leq 15 />15)$ & $93(50) / 93(50)$ \\
\hline \multicolumn{2}{|l|}{ Lymph node ratio } \\
\hline$(<0.05 />0.05)$ & $119(64) / 67(36)$ \\
\hline$(<0.1 />0.1)$ & $140(75) / 46(25)$ \\
\hline$(<0.2 />.0 .2)$ & $158(87) / 24(13)$ \\
\hline$(<0.3 />0.3)$ & $176(95) / 10(05)$ \\
\hline \multicolumn{2}{|l|}{ Recurrence } \\
\hline Present & $72(39)$ \\
\hline Absent & $114(61)$ \\
\hline \multicolumn{2}{|l|}{ Log-ODDS (LODDS) } \\
\hline $3 / 4 / 5$ & $107 / 73 / 06$ \\
\hline
\end{tabular}

levels of LNR and LODDS are shown in Table 4. The sensitivity for predicting DSS decreases with increasing LNR and LODDS thereby undermining its significance.

\section{Factors affecting Recurrence free survival (RFS)}

Univariate analysis revealed the $\mathrm{T}$ stage, tumor differentiation, lymph node positivity, LNR $(0.05,0.1)$, number of positive lymph nodes $(0,1-3,>3)$, LODDS and perineural invasion as significant risk factors influencing RFS (Table 3). On multivariate analysis, RFS was influenced by the T-stage and tumor differentiation (Table 3). Survival curves stratified as per T-stage, PNI, LODDS, differ- 
Table 2. Patients and tumor characteristics and their respective 3-year and 5-year survival for disease specific survival and recurrence free survival

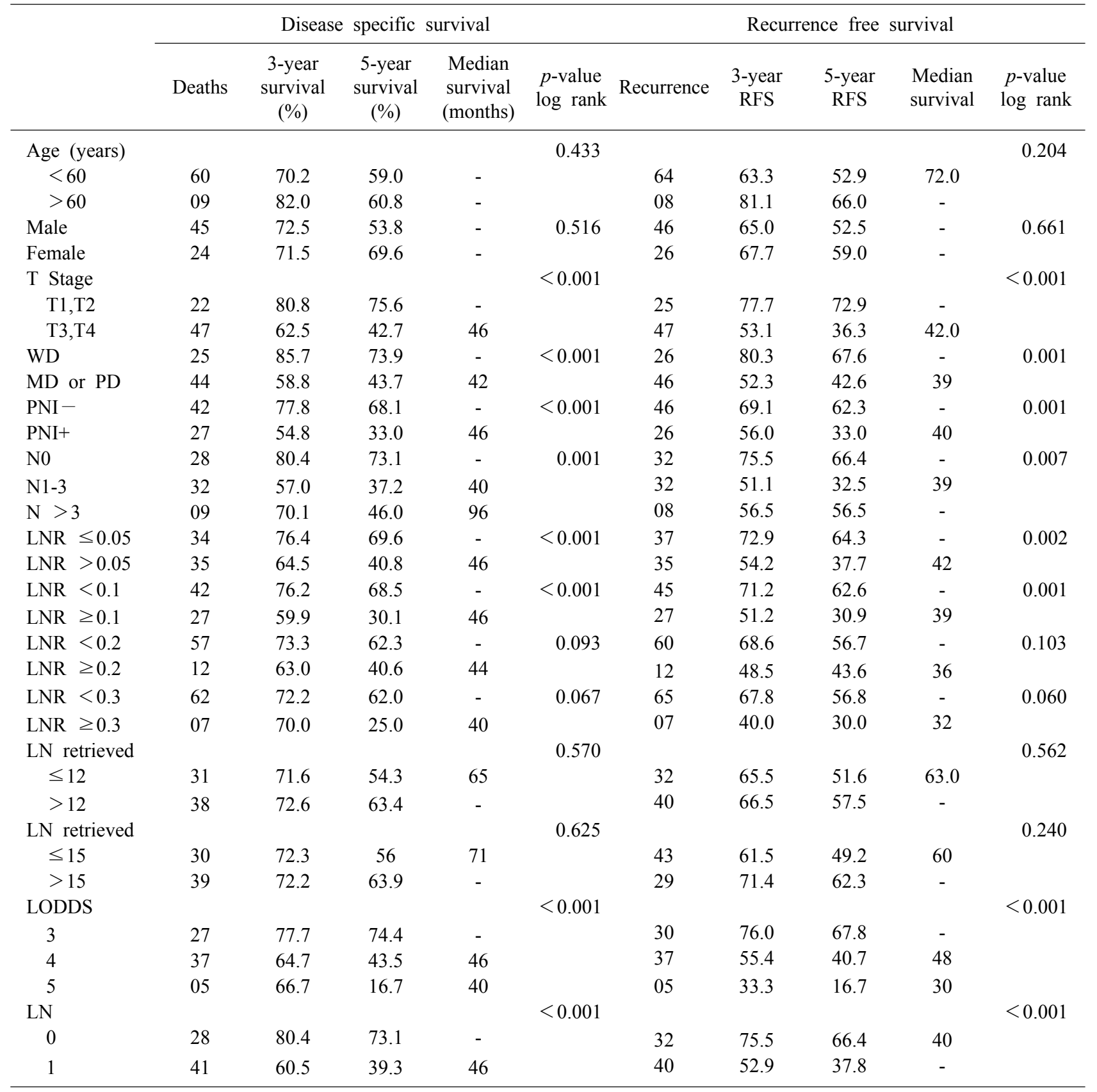

WD, well differentiated; MD, moderately differentiated; PD, poorly differentiated; PNI, perineural invasion; LN, lymph node; LNR, lymph node ratio

entiation, LNR (0.1), and number of positive lymph nodes (AJCC $8^{\text {th }}$ ed.) are shown in Fig. 3 for RFS. AUC of LNR and LODDS for predicting RFS (Fig. 3) was 0.629 $(p=0.003)$ and $0.677(p=0.001)$ respectively. The sensitivity of cut off levels decreases further with increase in LNR and LODDS thereby reducing its importance (Table 4).

\section{DISCUSSION}

Pancreatoduodenectomy remains the mainstay of treatment for patients with periampullary carcinoma, since it has the potential to offer cure and provide long-term survival. We report 59.3\% 5-year DSS and 54.9\% 5-year RFS in patients with ampullary variant of periampullary 


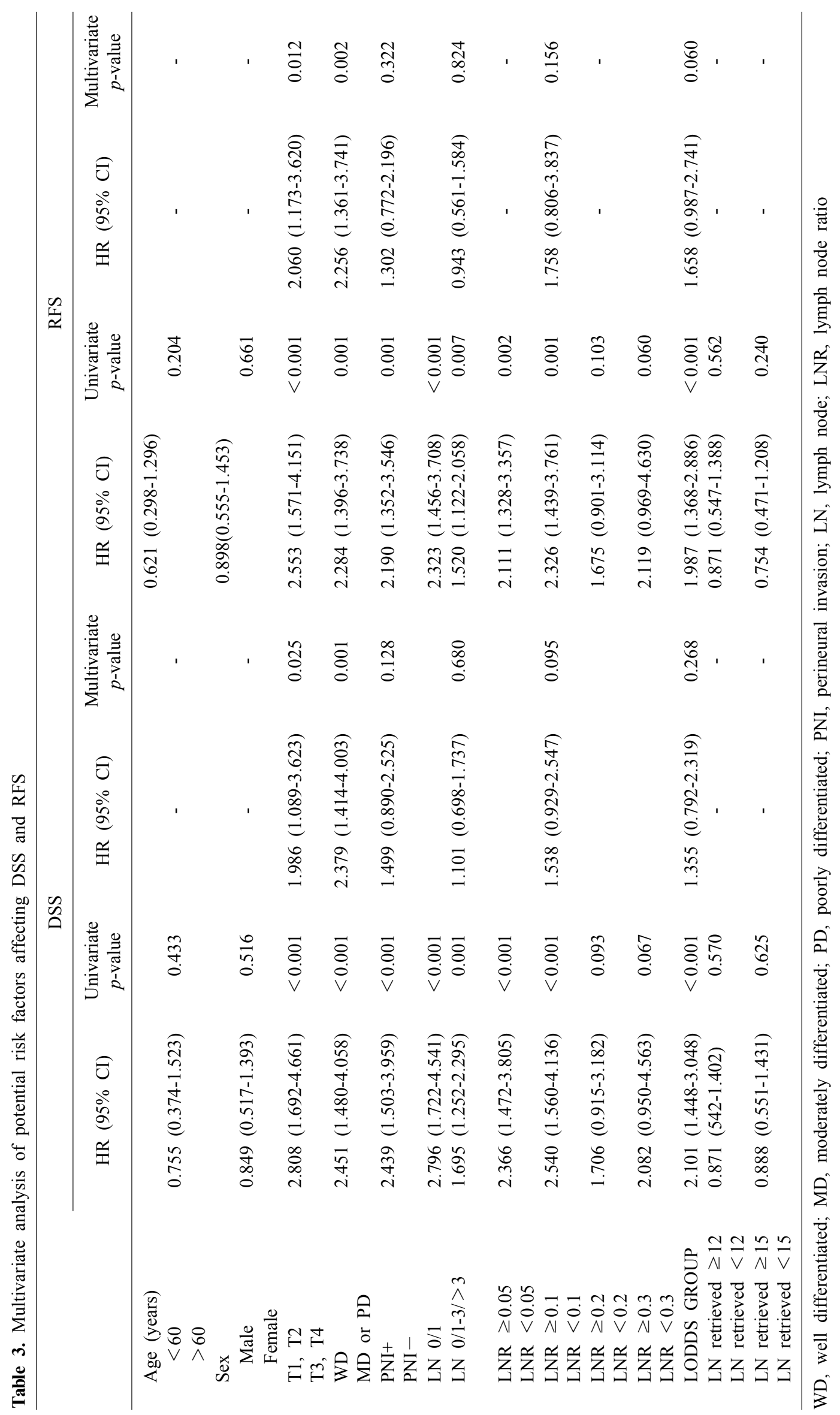



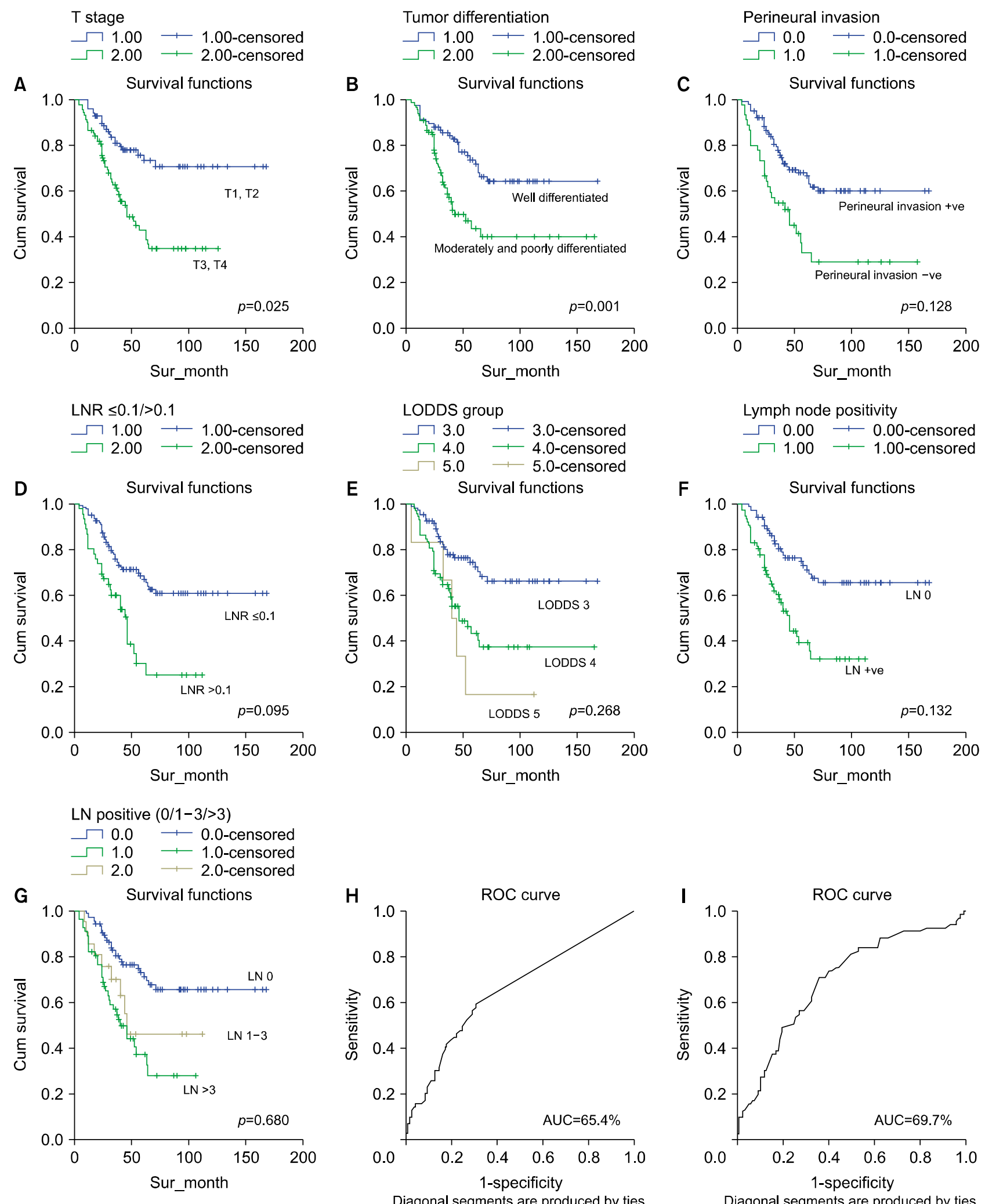

Fig. 2. (A and B) Depicting significantly better disease specific survival (DSS) in patients with T1/T2 stage and well differentiated ampullary tumors; (C-G) DSS is worse with patients with Perineural invasion, lymph node ratio (LNR) of $>0.1$, LODDS cut off at $4 \& 5$, lymph node positive disease, and $>3$ L.N positive, however not statistically significant; (H) ROC curves showing DSS prediction by LNR. AUC was 0.654 for DSS with $p$-value of $<0.001$; (I) ROC curves showing DSS prediction by LODDS. AUC was 0.697 for DSS with $p$-value of $<0.001$. 
Table 4. Sensitivity and specificity of LNR and LODDS ratio for predicting DSS and RFS (from ROC curve)

\begin{tabular}{ccccc}
\hline \multirow{2}{*}{ LNR } & \multicolumn{2}{c}{ DSS } & \multicolumn{2}{c}{ RFS } \\
\cline { 2 - 5 } & Sensitivity & Specificity & Sensitivity & Specificity \\
\hline-1.96 & 37.7 & 83.8 & 36.1 & 83.3 \\
-1.00 & 18.8 & 91.5 & 18.1 & 91.2 \\
-0.49 & 10.1 & 97.4 & 9.7 & 97.4 \\
& \multicolumn{2}{c}{ AREA- 0.654} & \multicolumn{2}{c}{ AREA- 0.629} \\
\hline \multirow{2}{*}{ LODDS } & \multicolumn{3}{c}{ DSS } & \multicolumn{3}{c}{ RFS } \\
ratio & Sensitivity & Specificity & Sensitivity & Specificity \\
\hline-1.96 & 100 & 9 & 100 & 9 \\
-1.00 & 62.3 & 67.5 & 59.7 & 66.7 \\
-0.49 & 20.3 & 90.6 & 19.4 & 90.4 \\
& \multicolumn{2}{c}{ AREA- 0.697} & AREA- & 0.677 \\
\hline
\end{tabular}

carcinoma undergoing curative resection. The better survival is similar to that reported by Sakata et al. which is $64 \%$ at 5 years but significantly higher when compared to Farid et al. (5 year actuarial survival $27 \%)^{10}$ and Lee et al. (median survival 28-31 months). ${ }^{9,12}$ The rationale behind the better survival in our series could be attributed two folds. Firstly, there was only ampullary tumors in our analysis, which are essentially known to have a better survival whereas pancreatic head tumors with their dismal prognosis were higher in the studies by Lee et al. ${ }^{9}$ and Farid et al. ${ }^{10,19}$ Secondly, the number of patients with lymph node positive disease (41\%) in our study was lower as compared to that reported by Farid et al. (68\%), ${ }^{10}$ Hurtuk et al. $(62 \%),{ }^{20}$ Hatzaras et al. $(58 \%)^{21} \&$ Falconi et al. $(50 \%)^{22}$

T-stage and tumor differentiation had significant impact on DSS \& RFS in both univariate and multivariate analysis. Apart from this, patients with T3/T4 disease had significant recurrence as compared to $\mathrm{T} 1 / \mathrm{T} 2$ disease ( $p$ $<0.001)$. The negative impact of tumor differentiation has been reported by Kim et al. ${ }^{23}$ and Doepker et al. ${ }^{24}$ as well. The various aspects of lymph nodes staging system such as LN positivity, number of LN retrieved, number of positive lymph nodes (N0, N1 and N2) (AJCC $8^{\text {th }}$ ed.), LNR and LODDS needs to be examined to assess the actual impact of lymph node involvement on the prognosis of ampullary carcinoma.

Lymph node positivity has been reported as a marker of poor prognosis in patients undergoing pancreatoduodenectomy. ${ }^{2,21,25,26}$ Pancreatic head tumors had been the preponderant tumors in the above-mentioned studies. However, in our study, LN positivity failed to reach statistical significance for DSS \& RFS on multivariate analysis and our results are in conformity with Farid et al. ${ }^{10} \&$ Choi et al. ${ }^{27}$ Doepker et al. ${ }^{24}$ in their study on 106 ampullary tumors found LN positivity not to be significant predictor for overall survival but was significant in predicting recurrence on multivariate analysis.

Patients with N1 and N2 positive lymph nodes had poor DSS and RFS on univariate analysis but it failed to reach statistical significance on multivariate analysis. Sakata et al. ${ }^{12}$ demonstrated that presence of $\geq 4$ positive LN predicted the outcome better than LN ratio for ampullary cancers. The significance of the number of positive lymph nodes, is still moot, though it's relevance has been reported by some authors in the past. ${ }^{27,28} \mathrm{Kang}$ et al. ${ }^{29}$ reviewed more than 1000 patients with ampullary tumors and proposed the modification in nodal staging according to number of metastatic LN into 3 tier. Consequently, in the current TNM staging system for ampullary carcinoma metastatic lymph nodes have been sub classified on the basis of their number. We classified the number of positive $\mathrm{LN}$ retrieved as per AJCC $8^{\text {th }}$ classification but did not find it consequential in multivariate analysis.

The effect of number of lymph nodes retrieved on long-term survival has also been studied with some support in literature. ${ }^{22,28}$ However, our study including the one by Farid et al. ${ }^{10}$ did not find total lymph node yield to be of any prognostic significance. Number of retrieved lymph nodes when stratified into N0 or N1 disease, did not affect survival, whether the cut-off level was kept at 12 or 15 lymph nodes. This though, does not diminish the importance of maximal lymph node retrieval; as adequate lymph node removal is still essential for proper staging of the disease besides eluding the stage migration effect.

Previous studies have tried to validate LNR as a categorical variable by either using cut-off levels ${ }^{10,25}$ or by grouping them. ${ }^{4,9,20,22}$ Farid et al. ${ }^{10} \&$ Shamseddine et al. ${ }^{28}$ found the LNR $>0.2$ to be an important factor in determining prognosis. The study by Shamseddine et al. ${ }^{28}$ was limited by the small sample size $(n=80)$ \& a low lymph node yield (median $n=9$ ). Pawlik et al., ${ }^{4}$ Lee et al., ${ }^{9}$ Hurtuk et al. ${ }^{20} \&$ Falconi et al. ${ }^{22}$ found that as the LNR groups increased, the survival decreased proportionately. While Sakata et al., ${ }^{12}$ Sierzega et al. ${ }^{13}$ and Murakami et 

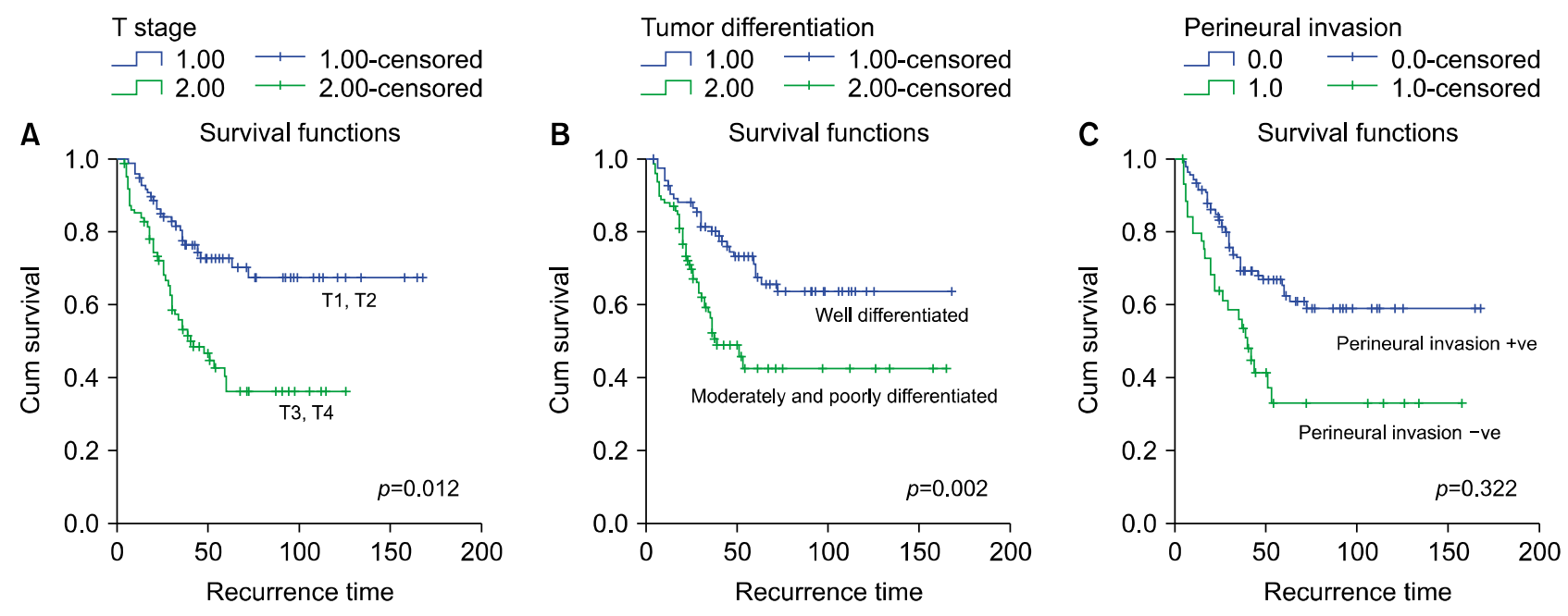

$\mathrm{LNR} \leq 0.1 />0.1$
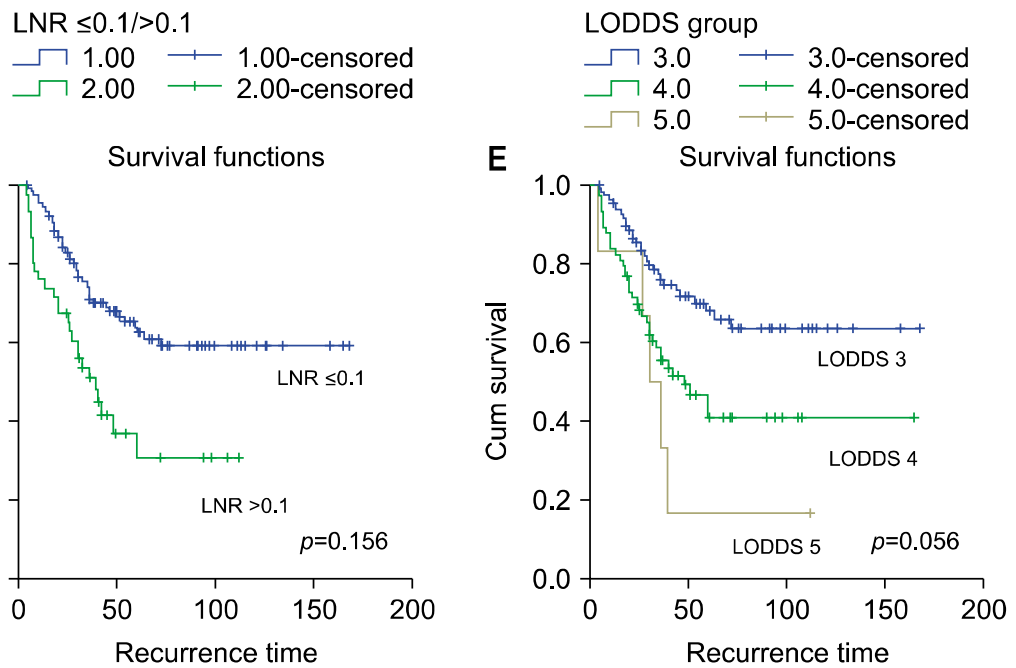

Lymph node positivity

$\neg 0.00 \longrightarrow 0.00$-censored

$\neg 1.00 \longrightarrow 1.00$-censored
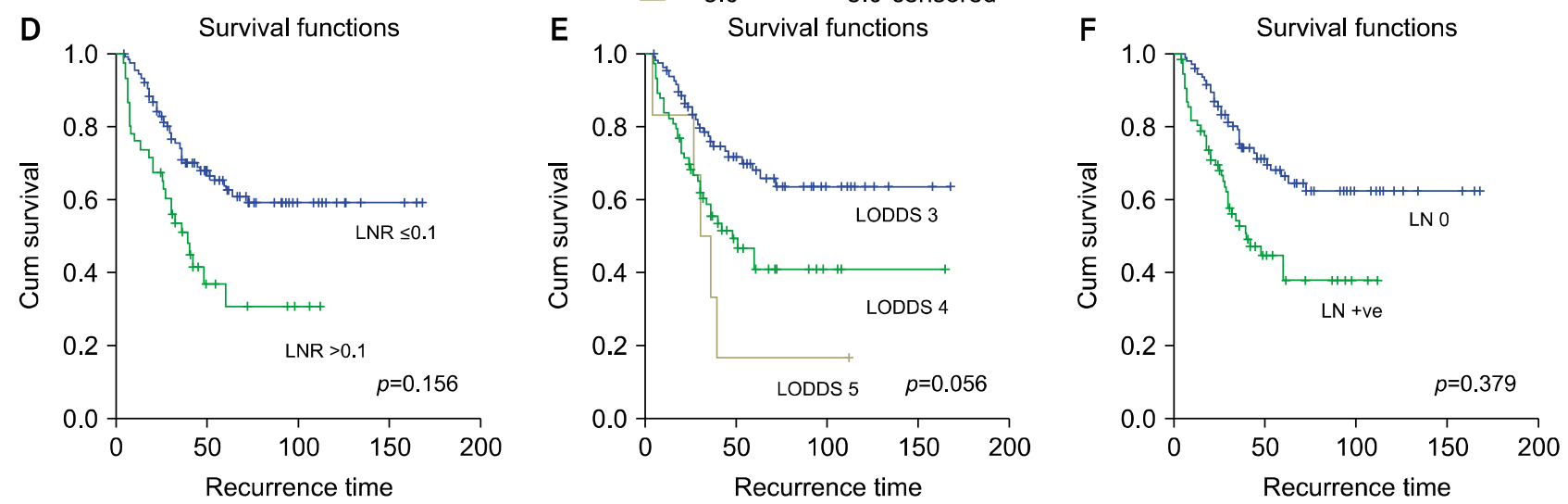

LN positive $(0 / 1-3 />3)$
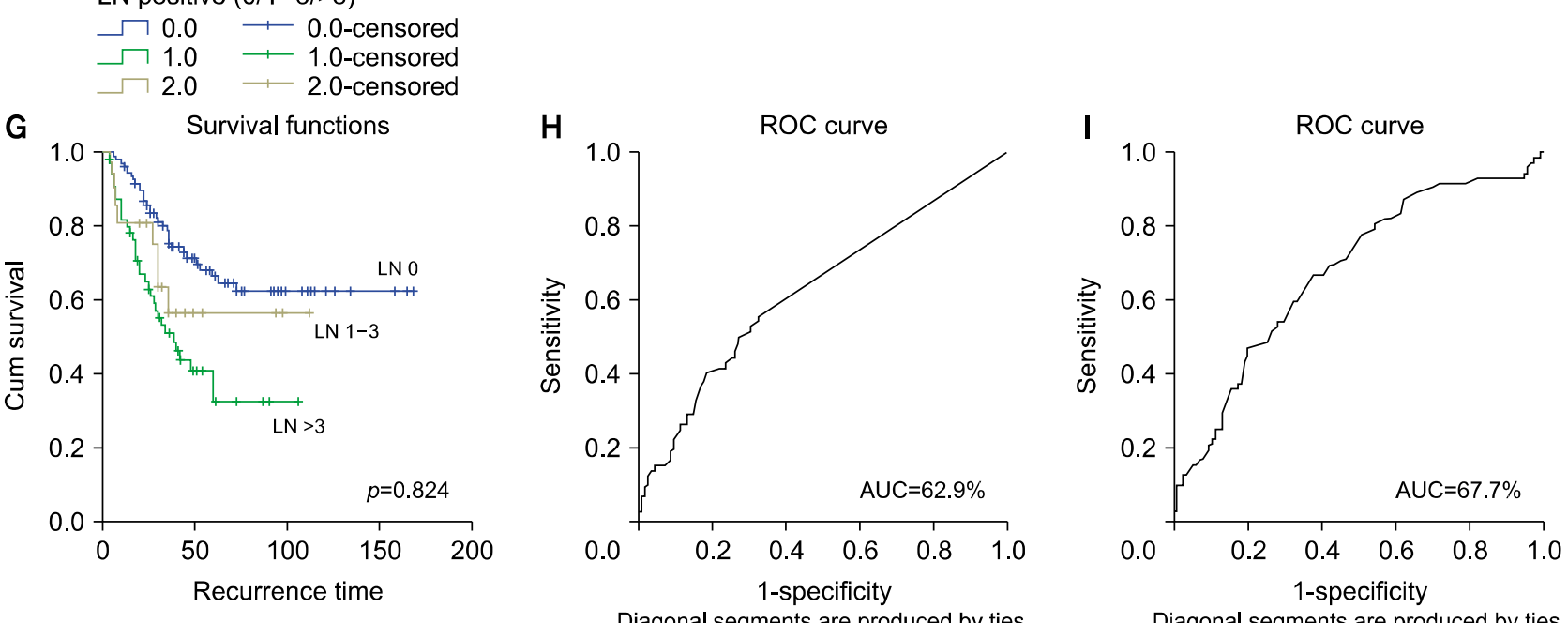

Fig. 3. (A and B) Depicting significantly lower recurrence free survival (RFS) in patients with T1/T2 stage and well differentiated ampullary tumor; (C-G) RFS is worse in patients with Perineural invasion, lymph node ration of $>0.1$, LODDS cut off at 4 \& 5, lymph node positivity, and $>3$ L.N. positive, however not statistically significant; (H) ROC curves showing RFS prediction by LNR. AUC was 0.629 for RFS with $p$-value of 0.003 ; (I) ROC curves showing RFS prediction by LODDS. AUC was 0.677 for RFS with $p$-value of $<0.001$. 
al. ${ }^{30}$ have dissuaded its use as a prognostic marker, Sakata et al. ${ }^{12}$ suggested that LNR may be useful when the median lymph node yield is low, as these patients may not have been adequately staged. We found LNR cut-off levels of 0.1 as having highest chi- square value amongst the LNR strata and a good predictor of RFS ( $p=0.001)$ \& DSS $(p=0.001)$ but failed to show any statistical significance on multivariate analysis whereas Doepker et al. ${ }^{24}$ also found LNR of 0.1 as significant predictor for OS and RFS in univariate analysis. We aver that since the number of lymph node involved is a continuous variable, it would be apt to analyze it as a continuous variable using ROC curve rather as a categorical variable. LNR with cut-off value of 0.1 showed a low sensitivity (DSS-37.7\%, RFS$36.1 \%$ ) \& low specificity (DSS-83.8\%, RFS-83.3\%), with AUC of 0.654 for DSS $(p<0.001) \& 0.629$ for RFS $(p=0.003)$. Though LNR has shown a low sensitivity \& specificity and a smaller AUC, $p$-values were significant for prediction of DSS \& RFS. House et al. ${ }^{8}$ demonstrated that $\mathrm{LN}$ ratio $>0.18$ was a strong predictor of survival in pancreatic cancer when evaluated as continuous variable while Berger et al. $^{31}$ reported that statistical significance was lost when LNR was examined as a continuous variable $(p=0.06)$. Tol et al. ${ }^{11}$ showed that LNR was a common predictor of poor survival for CBD, ampullary and pancreatic cancer.

Further, He et al. ${ }^{16}$ analyzed a total of 205 patients undergoing surgical resection for periampullary adenocarcinoma. In their study LODDS was able to stratify patients into various subgroups with significant differences of both DSS and RFS and based on the ROC curves, LODDS outperformed LNR and other LN staging systems in predicting DSS and RFS. In our study the predictive power of LODDS and LNR was almost the same for DSS, AUC being 0.697 and 0.677 for DSS and RFS respectively. However, in multi-variate analysis, both the lymph node stratifications did not reach statistical significance. Morales-Oyarvide et al. ${ }^{15}$ evaluated 600 patients who underwent surgical resection for pancreatic ductal adenocarcinoma (both head and body tail) and concluded that Lymph node ratio and LODDS were associated with a stepwise increase in the hazards for recurrence (Ptrend 1/4 0.004 and 0.005 , respectively). In this study, $49 \%$ patients had $\mathrm{R} 1 / \mathrm{R} 2$ resection, including both head \& body tail tumors vis a vis our study where in only patients under- going PD were ampullary.

Our study is limited by the retrospective analysis of data and a non-inclusion of few variables due to missing data in some patients. These include specific histologic subtype intestinal or pancreatobiliary variant and data related administration of adjuvant therapy especially in earlier patients in our series. However, the strengths of our study are the inclusion of both RFS and DSS in the analysis. Secondly, we evaluated LNR both as a continuous and categorical variable, and LODDS unlike most other studies that have either not examined RFS or have studied LNR solely as a categorical variable.

In conclusion, lymph node positivity and the number of positive nodes in ampullary tumors, unlike other abdominal malignancies lose their significance as a predictor of survival on multivariate analysis. LNR when analyzed as a continuous variable is able to predict DSS and RFS but when analyzed as a categorical variable, fails to show similar significance. Further studies are required to validate this concept since the sensitivity and specificity of LNR and LODDS for ampullary tumors is low.

\section{CONFLICT OF INTEREST}

None of the authors have any potential conflicts to disclose.

\section{ORCID}

Sundeep Singh Saluja:

https://orcid.org/0000-0003-2733-7204

Pramod Kumar Mishra:

https://orcid.org/0000-0003-0005-4044

Shashi Kiran: https://orcid.org/0000-0001-8148-6505

Harsh J. Shah: https://orcid.org/0000-0002-1184-7261

Sandip Chandrasekar: https://orcid.org/0000-0001-6097-8754

Mohammed Nayeem: https://orcid.org/0000-0003-1800-0001

Aditya Sharma: https://orcid.org/0000-0002-6248-5360

Vaibhav Kumar Varshney: https://orcid.org/0000-0003-1771-2787 


\section{AUTHOR CONTRIBUTIONS}

\author{
Conceptualization: SSS, PKM, HJS, SC, MAN. Data \\ curation: SSS, SK, HJS, SC, AS, VKV. Formal analysis: \\ SK, HJS, SC, AS, VKV. Methodology: SSS, PKM, HJS, \\ SC, MAN. Project administration: SSS, PKM, HJS, SC, \\ AS. Visualization: SSS, PKM, SC, MAN, VKV. Writing - \\ original draft: HJS, SC, AS. Writing - review \& editing: \\ SSS, PKM, SK, MAN, VKV.
}

\section{REFERENCES}

1. Albores-Saavedra J, Schwartz AM, Batich K, Henson DE. Cancers of the ampulla of Vater: demographics, morphology, and survival based on 5,625 cases from the SEER program. J Surg Oncol 2009; 100:598-605.

2. Sugimoto K, Sakamoto K, Tomiki Y, Goto M, Kotake K, Sugihara K. Proposal of new classification for stage III colon cancer based on the lymph node ratio: analysis of 4,172 patients from multi-institutional database in Japan. Ann Surg Oncol 2015;22:528-534.

3. Peschaud F, Benoist S, Julié C, Beauchet A, Penna C, Rougier $\mathrm{P}$, et al. The ratio of metastatic to examined lymph nodes is a powerful independent prognostic factor in rectal cancer. Ann Surg 2008;248:1067-1073.

4. Pawlik TM, Gleisner AL, Cameron JL, Winter JM, Assumpcao $\mathrm{L}$, Lillemoe KD, et al. Prognostic relevance of lymph node ratio following pancreaticoduodenectomy for pancreatic cancer. Surgery 2007; 141:610-618

5. Bhamidipati CM, Stukenborg GJ, Thomas CJ, Lau CL, Kozower BD, Jones DR. Pathologic lymph node ratio is a predictor of survival in esophageal cancer. Ann Thorac Surg 2012;94:1643-1651.

6. Marchet A, Mocellin S, Ambrosi A, de Manzoni G, Di Leo A, Marrelli D, et al; Italian Research Group for Gastric Cancer Study (GIRCG). The prognostic value of N-ratio in patients with gastric cancer: validation in a large, multicenter series. Eur J Surg Oncol 2008;34:159-165.

7. Slidell MB, Chang DC, Cameron JL, Wolfgang C, Herman JM, Schulick RD, et al. Impact of total lymph node count and lymph node ratio on staging and survival after pancreatectomy for pancreatic adenocarcinoma: a large, population-based analysis. Ann Surg Oncol 2008;15:165-174.

8. House MG, Gönen M, Jarnagin WR, D’Angelica M, DeMatteo $\mathrm{RP}$, Fong $\mathrm{Y}$, et al. Prognostic significance of pathologic nodal status in patients with resected pancreatic cancer. J Gastrointest Surg 2007;11:1549-1555.

9. Lee SR, Kim HO, Park YL, Shin JH. Lymph node ratio predicts local recurrence for periampullary tumours. ANZ J Surg 2014;84: 353-358.

10. Farid SG, Falk GA, Joyce D, Chalikonda S, Walsh RM, Smith $\mathrm{AM}$, et al. Prognostic value of the lymph node ratio after resection of periampullary carcinomas. HPB (Oxford) 2014;16: 582-591.

11. Tol JA, Brosens LA, van Dieren S, van Gulik TM, Busch OR, Besselink MG, et al. Impact of lymph node ratio on survival in patients with pancreatic and periampullary cancer. Br J Surg 2015; 102:237-245.

12. Sakata J, Shirai Y, Wakai T, Ajioka Y, Akazawa K, Hatakeyama $\mathrm{K}$. Assessment of the nodal status in ampullary carcinoma: the number of positive lymph nodes versus the lymph node ratio. World J Surg 2011;35:2118-2124.

13. Sierzega M, Nowak K, Kulig J, Matyja A, Nowak W, Popiela T. Lymph node involvement in ampullary cancer: the importance of the number, ratio, and location of metastatic nodes. J Surg Oncol 2009;100:19-24.

14. Böttger TC, Boddin J, Heintz A, Junginger T. Clinicopathologic study for the assessment of resection for ampullary carcinoma. World J Surg 1997;21:379-383.

15. Morales-Oyarvide V, Rubinson DA, Dunne RF, Kozak MM, Bui $\mathrm{JL}$, Yuan $\mathrm{C}$, et al. Lymph node metastases in resected pancreatic ductal adenocarcinoma: predictors of disease recurrence and survival. Br J Cancer 2017;117:1874-1882.

16. He C, Mao Y, Wang J, Huang X, Lin X, Li S. Surgical management of periampullary adenocarcinoma: defining an optimal prognostic lymph node stratification schema. J Cancer 2018;9:16671679.

17. Ramacciato G, Nigri G, Petrucciani N, Pinna AD, Ravaioli M, Jovine E, et al. Prognostic role of nodal ratio, LODDS, $\mathrm{pN}$ in patients with pancreatic cancer with venous involvement. BMC Surg 2017;17:109.

18. Mishra PK, Saluja SS, Gupta M, Rajalingam R, Pattnaik P. Blumgart's technique of pancreaticojejunostomy: an appraisal. Dig Surg 2011;28:281-287.

19. Chen SC, Shyr YM, Wang SE. Longterm survival after pancreaticoduodenectomy for periampullary adenocarcinomas. HPB (Oxford) 2013;15:951-957.

20. Hurtuk MG, Hughes C, Shoup M, Aranha GV. Does lymph node ratio impact survival in resected periampullary malignancies? Am J Surg 2009;197:348-352.

21. Hatzaras I, George N, Muscarella P, Melvin WS, Ellison EC, Bloomston M. Predictors of survival in periampullary cancers following pancreaticoduodenectomy. Ann Surg Oncol 2010;17: 991-997.

22. Falconi M, Crippa S, Domínguez I, Barugola G, Capelli P, Marcucci S, et al. Prognostic relevance of lymph node ratio and number of resected nodes after curative resection of ampulla of Vater carcinoma. Ann Surg Oncol 2008;15:3178-3186.

23. Kim RD, Kundhal PS, McGilvray ID, Cattral MS, Taylor B, Langer B, et al. Predictors of failure after pancreaticoduodenectomy for ampullary carcinoma. J Am Coll Surg 2006;202:112-119.

24. Doepker MP, Thompson ZJ, Centeno BA, Kim RD, Wong J, Hodul PJ. Clinicopathologic and survival analysis of resected ampullary adenocarcinoma. J Surg Oncol 2016;114:170-175.

25. Norero E, Viñuela E, Báez S, Martínez C, Reyes J, Kusanovic $\mathrm{R}$, et al. [Results of pancreaticoduodenectomy in the treatment of periampullary tumors]. Rev Med Chil 2011;139:1015-1024. Spanish.

26. Jarufe NP, Coldham C, Mayer AD, Mirza DF, Buckels JA, Bramhall SR. Favourable prognostic factors in a large UK experience of adenocarcinoma of the head of the pancreas and periampullary region. Dig Surg 2004;21:202-209.

27. Choi SB, Kim WB, Song TJ, Suh SO, Kim YC, Choi SY. Surgical outcomes and prognostic factors for ampulla of Vater cancer. Scand J Surg 2011;100:92-98.

28. Shamseddine AI, Mukherji D, Melki C, Elias E, Eloubeidi M, Dimassi H, et al. Lymph node ratio is an independent prognostic factor after resection of periampullary malignancies: data from a tertiary referral center in the middle East. Am J Clin Oncol 2014;37:13-18.

29. Kang HJ, Eo SH, Kim SC, Park KM, Lee YJ, Lee SK, et al. Increased number of metastatic lymph nodes in adenocarcinoma of the ampulla of Vater as a prognostic factor: a proposal of new nodal classification. Surgery 2014;155:74-84. 
30. Murakami Y, Uemura K, Sudo T, Hayashidani Y, Hashimoto Y, Nakashima A, et al. Number of metastatic lymph nodes, but not lymph node ratio, is an independent prognostic factor after resection of pancreatic carcinoma. J Am Coll Surg 2010;211:196204.
31. Berger AC, Watson JC, Ross EA, Hoffman JP. The metastatic/ examined lymph node ratio is an important prognostic factor after pancreaticoduodenectomy for pancreatic adenocarcinoma. Am Surg 2004;70:235-240; discussion 240. 